\title{
The Design of SoS Multi-Agent System Model Based on Emergence Imitation
}

\author{
Qiang Qu, Xinhua He, Hao Cheng and Weichao Zhang \\ Department of Information Engineering, Academy of Army Armored Force, Beijing 100072, China
}

\begin{abstract}
The traditional modeling method of multi-Agent system (MAS) for weapon systems is to build the underlying Agent unit in micro perspective, and then integrate the units to make a larger system. The MAS built by this way cannot show the emerged characteristics of the macro system in the simulation, the effectiveness has been questioned. To solve this problem, the traditional weapon system Agent modeling method has been improved, to put forward an emergence-imitation-based MAS modeling method. Firstly, the framework of Agent combat capability model is designed to meet the needs of system simulation. Then, a method of constructing Agent behavior model based on emergence is proposed. Lastly, the method is used to build an MAS model for an armored brigade. The simulation results demonstrate that the MAS model constructed by this method can produce emerged characteristics of real weapon systems in the simulation, which proved the effectiveness of the method.
\end{abstract}

Keywords-weapon equipment; SoS; emergence

\section{INTRODUCTION}

Weapon equipment systems, also called system of systems (SoS) is a higher level of large-scale system which consists of a variety of weapons equipment system by the function of mutual contact and interact ${ }^{[1]}$. Due to the large interaction between weapon equipment and its operators, it is difficult to characterize and study the weapon equipment system by static and analytic methods. Simulation method is considered to be the most effective method for weapon system analysis and evaluation, and the most representative one is the Agent-Based Modeling and Simulation (ABMS) method ${ }^{[2]}$ 。

The ABMS method uses a bottom-to-up, individual-towhole, micro-to-macro approach to study complex systems, and performs the overall performance of the system by constructing an Agent model element and describing interaction between units, or between units and environment. ABMS is the most promising method to study and solve the combat effectiveness evaluation of weapon equipment system. The U.S. Army used this method to build EINSTein, SEAS ${ }^{[3]}$, SWarrior, ACME ${ }^{[4]}$ and so on. Australia built CAVALIER, WISDOM $^{[5]}$ and so on, and New Zealand built MANA ${ }^{[6]}$ and so on.

However, the above systems also mainly stay in the traditional modeling simulation way based on Agent. Firstly, Agent behavior is relatively simple, such as MANA behavior model mainly based on the cellular automata with 50 states; Secondly, the Agent behavior model is built for the individual micro characteristics, not constructed for the macro characteristics of the system, and the model cannot reflect the emergence of SoS in combat. Luo Peng-cheng ${ }^{[7]}$ pointed out that behavior modeling is a difficult problem in the SoS combat simulation. This paper improves the traditional MAS modeling method of SoS by using the idea based on emergence imitation.

\section{The Traditional Modeling Method of MAS MODEL FOR WEAPON SYSTEMS}

MAS model is one kind of SoS models, which is based on the idea of Agent modeling, simulates weapon equipment in system by using Agent model with independent behavior logic, and then simulates SoS and its combat behavior by the interaction of a large number of Agent models. Different from other Agent systems, the Agent system of SoS contains more Agent, and focuses on the Combat behavior of Agent.

The basic ideas of traditional MAS model construction procedure for SoS is shown in Figure 1. Firstly, it analyzes microscopically the constituent elements of the system, establish respectively the Agent behavior model and capacity model, then encapsulate the both. Finally, according to the organizational structure model obtained by macro analysis, integrate a large number of Agent models into the weapon system MAS model.

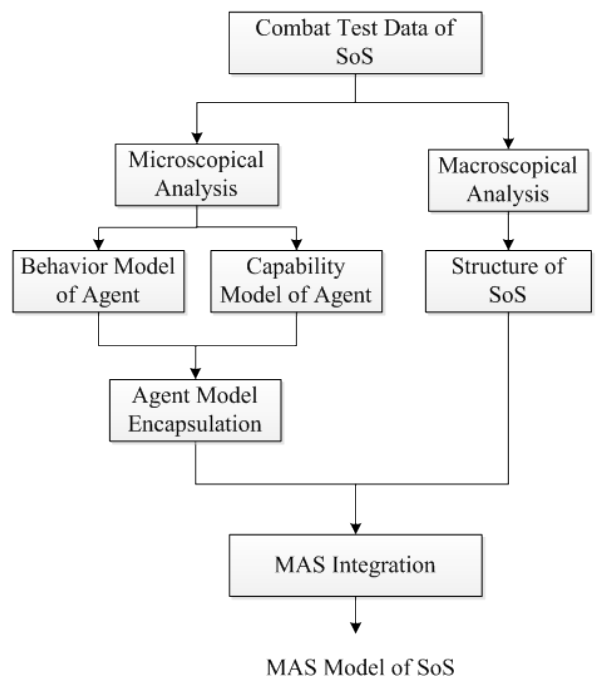

FIGURE I.TRADITIONAL MAS MODELING PROCEDURE FOR SOS

The traditional method of constructing MAS model mainly adopts the idea of "micro perspective modeling + macroscopic integration", lacks the modeling of the interaction among weapon equipment agents and does not reflect the emergence 
of the system. To solve this problem, the subject will use the related methods of micro-macro analysis, system dynamics and artificial intelligence to study the construction method of system model. The core idea of this method is "emergence imitation".

\section{MAS MOdel CONSTRUCTION METHOd OF WeAPON EQUIPMENT SYSTEM BASED ON EMERGENCE IMITATION}

\section{A. Method Principle and Process}

The modeling of weapon equipment system need depict micro behavior and attribute, but it is more important to simulate macro "emergence". There are many kinds of weapons, and the behavior and characteristics of them are complex. If getting together, it will form a variety of emergent phenomena and attributes. The MAS model construction method of weapon system based on emergence imitation is to model the Agent of weapon equipment around the simulation of macro emergence characteristics, and then to form the system model.

The method flow is shown in Figure 2. First of all, we need collect a large amount of weapons equipment (system) combat test data, and then make micro, macro-and-macro and macro analysis respectively to get Agent combat capability model, Agent combat behavior model and SoS structure model, and then do Agent model encapsulation and Agents integration to form the ultimate MAS model of SoS.

The dashed frame part is the Agent modeling content of weapon equipment which will be emphatically studied in this paper. The micro analysis is to observe and analyze the combat test data of weapon equipment (system) under a certain modeling granularity, and extract the Agent combat capability model. Before the micro-and-macro analysis, we need to first extract weapon system combat emergence, then analyze the mapping relationship between macro combat emergence and micro agent behaviors, and last construct the agent combat behavior model. The Agent model of weapon equipment is obtained by encapsulating the Agent combat capability model and the combat behavior model. It can be seen that the extraction of combat emergent features of weapon equipment is the premise and foundation of the Agent model construction. This paper assumes that the combat emergence features are known, and do not discuss it in detail.

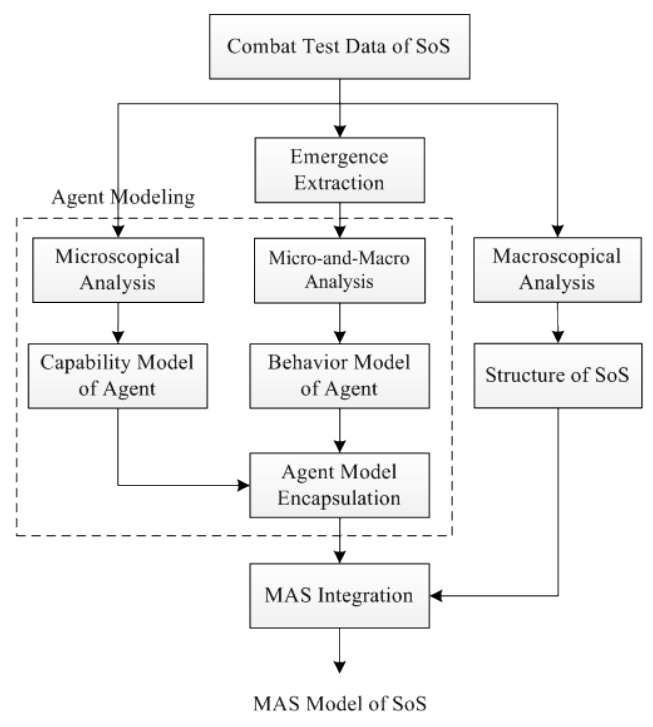

FIGURE II.

THE MAS MODELING PROCESS FOR SOS BASED ON EMERGENCE IMITATION

The subject of Agent modeling is the combat unit combining man with equipment. There are two meaning need to pay attention. First of all, It cannot be just cold equipment, must be the combination of man and equipment. Secondly, it cannot be, must be a unit with attack ability other than a noncombat or combat auxiliary unit. According to the granularity, the subject of Agent modeling can be divided into the single equipment, the platoon equipments, the company equipments, the battalion equipments and so on, hereinafter referred to as the weapon equipment Agent modeling.

Agent basic model based on automata is adopted in this paper. Automata was originally a robot that was proposed to imitate the behavior of humans and animals, which has now been abstracted into a class of models that can be implemented with physical machines or software code. The automata is defined as the subject model with finite state and fixed behavior rule base, and the corresponding relation between structure and function is shown in Figure 3. In automata structure, the logic engine and the state memory unit are implemented by some simple logical judgment statements. The behavior rules, the perception unit and the execution unit are the main contents of the modeling. The behavior rule set is defined as Agent behavior model, and the physical ability of execution unit and perception unit is defined as Agent capability model.

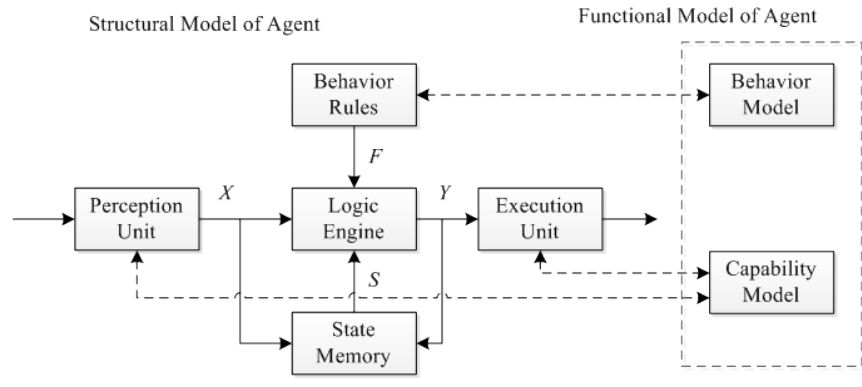

FIGURE III. MODEL BASED ON AUTOMATA 


\section{B. Framework Design of Agent Combat Capability Model}

The Agent capability model of weapon equipment refers to the index system which constitutes the combat capability. The index of the model must be oriented to the needs of system simulation, compatible with the Agent combat behavior model, and convenient for acquisition and integration.

Based on the above principles, the framework of Agent combat capability model for weapon equipment is designed, as shown in Figure 4. The framework of combat capability model is a basic framework, which is the foundation of Agent combat capability construction for weapon equipment. In practical application, other necessary capability indexes can also be added as needed.

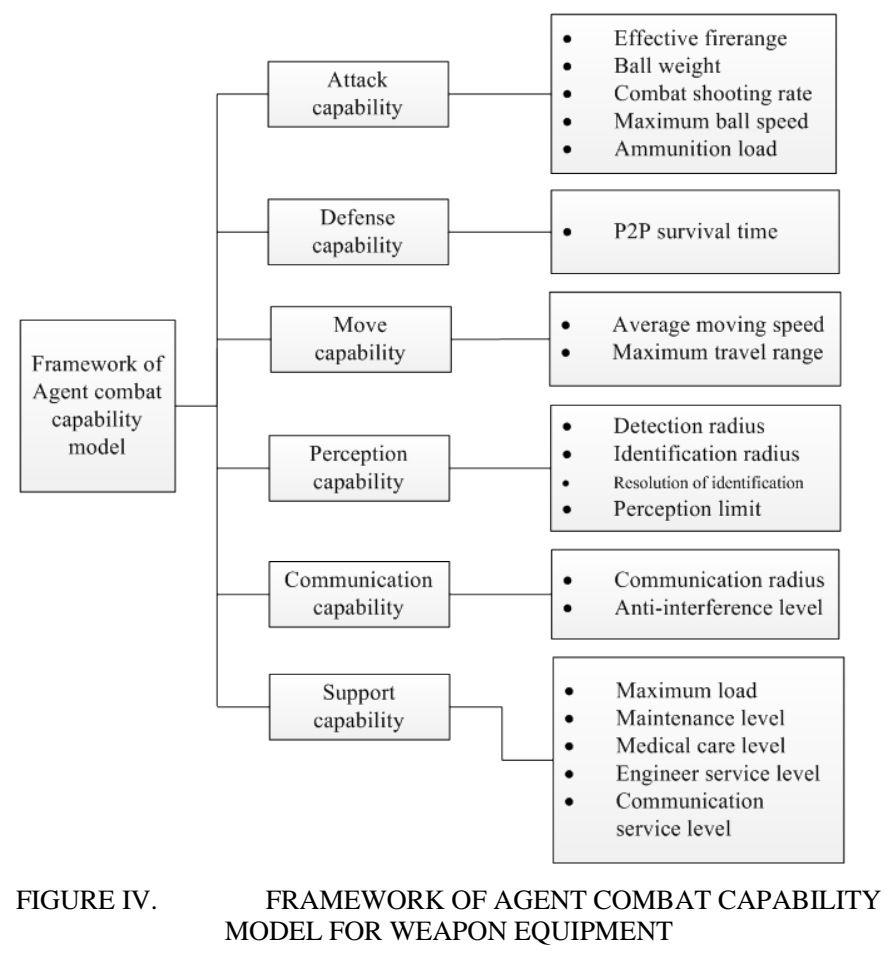

Ball weight, combat shooting rate and maximum speed of ball and other indicators of ammunition launch characterize attack ability. Among them, the ball weight refers to the warhead weight, not the whole bullet weight. Regardless of the difference in the explosive power of ammunition, the killer radius is largely determined by the Ball weight. Combat shooting rate refers to the launching speed under the operation of skilled soldiers. Combat shooting speed is usually less than the maximum shooting speed. The maximum speed of ammunition is defined as the theoretical maximum speed after the acceleration of ammunition. In order to meet need of attack behavior modeling and the synthetic comparison of attack capability, we combine the three indexes to define a new vector that is ammunition firepower. The direction of ammunition firepower is the direction of ammunition speed. The meaning of 1 unit of ammunition firepower is equivalent to a weapon accelerating $1 \mathrm{~kg}$ of ammunition from standstill to $1 \mathrm{~m} / \mathrm{s}$ within 1 second, which is roughly equivalent to the firepower of 1 ordinary handgun.
In order to facilitate the calculation, the ammunition firepower is defined as irrelevant to the ammunition type and the attacked object. This simplification can be accepted in the application of combat capability evaluation without considering the attack effect, but it is obviously not feasible in operational effectiveness evaluation. Therefore, we use a P2P parameter table to characterize the defense capability to make up for the deficiency of the ammunition firepower.

TABLE I. SURVIVAL TIME TABLE

\begin{tabular}{cccccc}
\hline & $\mathrm{B}_{1}$ & $\mathrm{~B}_{2}$ & $\cdots$ & $\mathrm{B}_{\mathrm{N}-1}$ & $\mathrm{~B}_{\mathrm{N}}$ \\
\hline $\mathrm{A}_{\mathrm{i}}$ & $D_{\mathrm{i}, 1}$ & $D_{\mathrm{i}, 2}$ & $\cdots$ & $D_{\mathrm{i}, \mathrm{N}-1}$ & $D_{\mathrm{i}, \mathrm{N}}$ \\
\hline
\end{tabular}

In the table, $D_{i, 1}, D_{i, 2}, \ldots D_{i, \mathrm{~N}}$ respectively denote the survival time of the $A_{i}$ against all enemy weapons $B_{j}(j=1,2, \ldots$, N).

The combat capability index is different from the technical and tactical indicators of the weapons equipment. It is an index directly reflecting combat capability, which is formed by a series of methods such as simplification and synthesis to meet the needs of combat behavior modeling and combat simulation. The data of combat capability indicators mainly come from weapons equipment combat tests, training exercises, simulation experiments, war history and war cases etc..

\section{Construction of Agent Combat Behavior Model}

The logic of behavior can be expressed in terms of rules. Rules refer to the laws and logic followed by the operation and operation law of things. In artificial intelligence, production rules are often used to represent knowledge and behavior generated by knowledge. The production rule consists of two parts, that is, the condition of the causality premise and the action of the causality result. The production rule is also called the Conditions-Action rule ( $\mathrm{C}$-A rule), its basic form is

$$
\mathrm{c} \rightarrow \mathrm{a} \text { or if } \mathrm{c} \text { then } \mathrm{a}
$$

Among them, "c" represents the condition, is the input of the rule; "a" represents the action, is the output of the rule. The conditions and actions of rule are usually more than one, and the general form is

$$
<\mathrm{C}, \mathrm{f}_{\mathrm{c}}(\cdot)>\rightarrow<\mathrm{A}, \mathrm{f}_{\mathrm{a}}(\cdot)>\text { or if }<\mathrm{C}, \mathrm{f}_{\mathrm{c}}(\cdot)>\text { then }<\mathrm{A}, \mathrm{f}_{\mathrm{a}}(\cdot)>
$$

Among them, $\mathbf{C}=\left\{\mathbf{c}_{1}, \mathbf{c}_{2}, \cdots, \mathbf{c}_{\mathbf{n}}\right\}$ represents the set of conditions, $f_{\mathrm{c}}()$ represents the composite formula of conditions, $\mathbf{A}=\left\{\mathbf{a}_{1}, \mathbf{a}_{2}, \cdots, \mathbf{a}_{\mathbf{m}}\right\}$ represents the set of actions, and $f_{\mathrm{a}}()$ represents the composite formula of actions. The above form is defined as a rule-based behavior model.

The construction of weapon equipment Agent behavior rule set based on emergence imitation is not to list all the actual behavior rules that the commanders of weapon equipment follow in combat, but to extract the most basic and simple rule 
set. This set of rules will, on the one hand, be able to generate the overall emergence behavior in the weapons system combat, on the other hand, be simple and refined without causing overload of simulation calculation.

According to the emerging conditions and dynamic mechanisms, the conditional acquisition unit must collect some system states that can be influenced by the actions of Agent behavior, so that the behavior rules may emerge. In SoS combat, we focus on a special system state called battlefield situation. The battlefield situation (referred to as the situation below) is defined as the global or partial state of both the enemy and ourselves as well as the environment during the combat. The elements of the situation include our troops deployment, our weapons equipment status and other factors, enemy forces deployment, the enemy's weapons equipment status and other enemy factors, road conditions, terrain, weather and other environmental factors ${ }^{[11]}$. We define the situation elements as the situations.

The number of situations for a single behavior is limited. Suppose that weapon Agent some behavior $\beta$ rule can be expressed as

$$
<\mathrm{C}^{\beta}, f_{\mathrm{B}^{\beta}}(\cdot)>\rightarrow<\mathrm{A}^{\beta}, f_{\mathrm{a}^{\beta}}(\cdot)>
$$

Among them, $\mathbf{C}^{\boldsymbol{\beta}}=\left\{\mathbf{c}_{1}, \mathbf{c}_{2}, \cdots, \mathbf{c}_{\mathbf{n}}\right\}$ represents the set of conditions for behavior $\beta, f_{\mathbf{e}^{\beta}}(\cdot)$ represents the composite formula of the condition, $A^{\mathbf{\beta}}=\left\{\mathbf{a}_{1}, \mathbf{a}_{2}, \cdots, \mathbf{a}_{\mathbf{m}}\right\}$ represents the set of actions, $f_{\mathrm{a}^{\mathrm{B}}}(\cdot)$ represents the composite formula of actions.

According to the rules of behavior defined constraints, each condition contains only one input state, so the behavior $\beta$ needs to collect $n$ kinds of input states $\mathbf{S}^{\beta-\text { in }}=\left\{s_{1}^{\beta \text { in }}, s_{2}^{\beta \text { in }}, \cdots, s_{n}^{\beta \text { in }}\right\}$, including the individual state, but also the system state. In the case of $n$ input states, there are $j$ species $(j \leq n)$ is the situation, and there are $k$ species $(k \leq j)$ state can be changed or affected by the action of behavior $\beta$, with state feedback loops as shown in Figure 5.

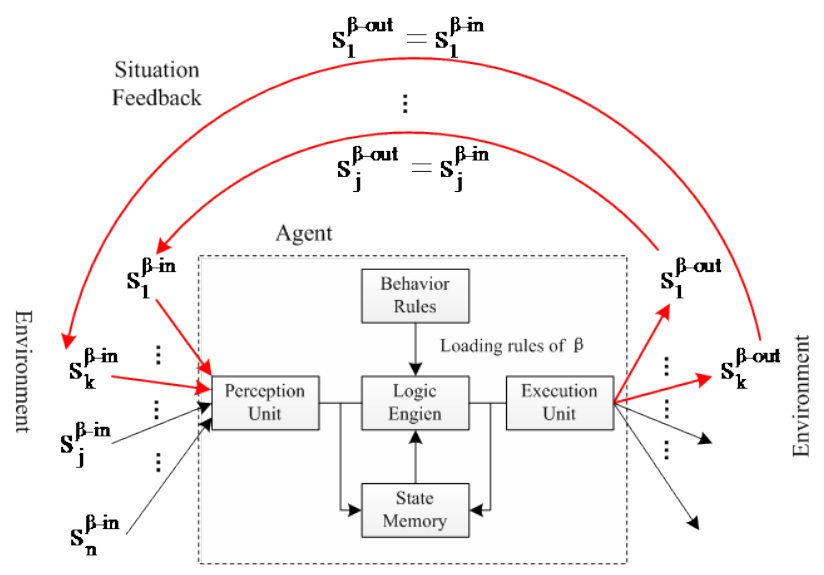

FIGURE V.

THE DIAGRAM OF SITUATION FEEDBACK
Situation feedback is the dynamic mechanism of emergence, and the core of emergence imitation is to simulate the feedback of situations. Feedback situation is the emergingforming bridge and is the dominant factor in the dynamic evolution of the system. In synergetic theory, it is also called the order parameter of the system. Other inputs except the feedback situation, both the individual state and the system state are equivalent to the stochastic disturbance in the evolution process of the whole system, and cannot be considered in the emergence imitation. In addition, the research shows that the same emergence comes from similar behaviors with some situation correlation, so it can further simplify the behavioral rule set which needs to be modeled. The behavior modeling process based on emergence imitation is shown in Figure 6.

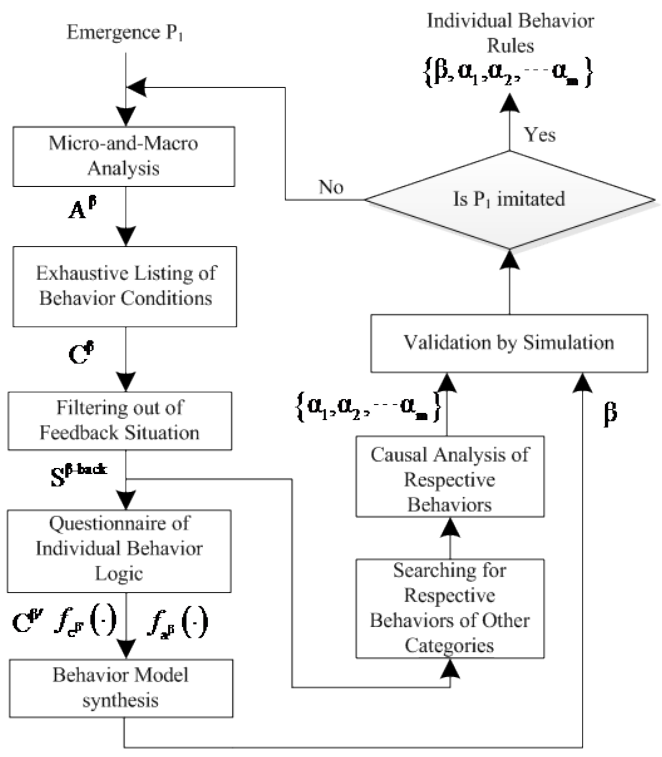

FIGURE VI.

THE BEHAVIOR MODELING PROCESS BASED ON EMERGENCE IMITATION

Firstly, micro-and-macro analysis is used to determine the action set $\mathbf{A}^{\boldsymbol{\beta}}$ of similar behavior. The conditional set $\mathbf{C}^{\boldsymbol{\beta}}$ in which elements are necessary when implementing $\beta$ behavior, is determine through exhaustive listing by all individuals. Then, we analyze the conditional state of each condition in the condition set, filter out the system-level state that can be changed by the $\beta$ behavior, which is incorporated into the feedback situation set, denoted as $\mathbf{S}^{\beta \text { bark }}=\left\{\mathrm{s}_{1}^{\beta \text { back }}, \mathbf{s}_{2}^{\beta \text { back }}, \cdots, \mathrm{s}_{\mathrm{n}}^{\beta \text { bark }}\right\}$. And it then search for behaviors of other categories related to each feedback situations, and carry out the system dynamics causal analysis to obtain the relevant behavioral model $\left\{\boldsymbol{\alpha}_{1}, \boldsymbol{\alpha}_{2}, \cdots \boldsymbol{\alpha}_{\mathrm{m}}\right\}$. At the same time, the questionnaire of individual behavior logic was conducted. With conditions of feedback situation as input and actions as output, the individual behavior logic questionnaire is made for all individuals to determine the condition set $\mathbf{C}^{\boldsymbol{\beta}^{\prime}}$, condition synthesis function $f_{\mathbf{c}^{\beta}}^{\prime}(\cdot)$ and action synthesis function $f_{\mathbf{a}^{\mathrm{B}}}(\cdot)$ of $\beta$ behavior. 


\section{SimUlation APPLICATION}

The relatively mature modeling simulation environment based on Agent includes NetLogo, Repast and Swarm. We choose the easy-to-use NetLogo environment, and use the Visual C + + as a supplement to NetLogo.

In NetLogo, this paper uses the article method to build the MAS model of American heavy brigade, which includes 58 tanks(such as M1A2), 58 armored vehicles (such as M113A3), 36 artilleries (such as M777), 500 infantry armed with anti-tank guns, rifles, machine guns and other types of firearms. Assuming that in the plains open area, the U.S. heavy brigade is encountered with another heavy combat team (named Blue Team). The number of Blue Team's equipments is similar to that of the U.S. heavy brigade, mainly including tanks, armored vehicles, vehicle mounted anti-tank guns, infantry, and etc.. it constitutes a positional defense situation, and adopts a simple defense attack strategy. The screenshot of the combat simulation process is shown in Figure 7. Eventually, the U.S. heavy brigade wiped out the Blue Team.

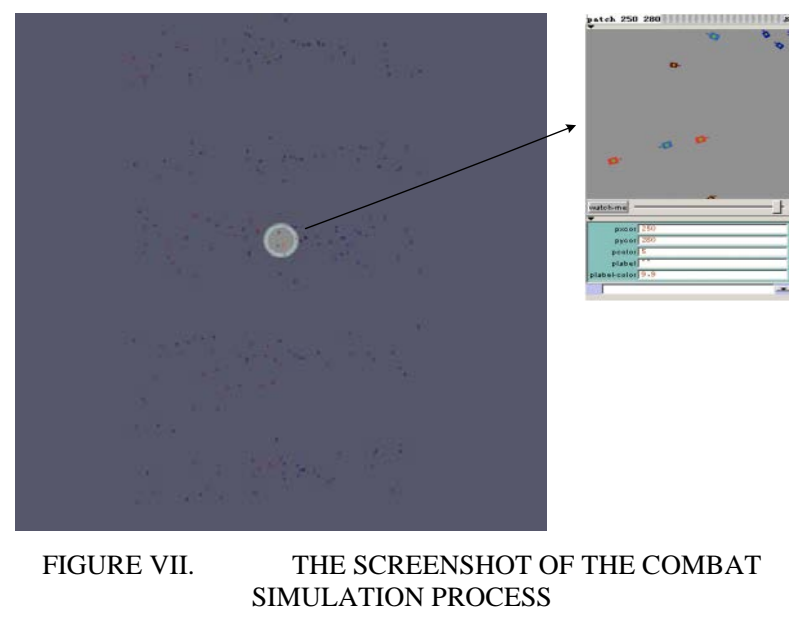

Through the comparison between the actual combat process and the combat simulation process, it is found that MAS model constructed by using the article method shows the characteristics of distribution of bombs to target, as shown in Figure 8, as well as the instantaneous distribution of the firing point under infantry and artillery coordination. Constrained by the research basis and conditions, it only simulates two emergent features, which is sufficient to prove the validity of the method.

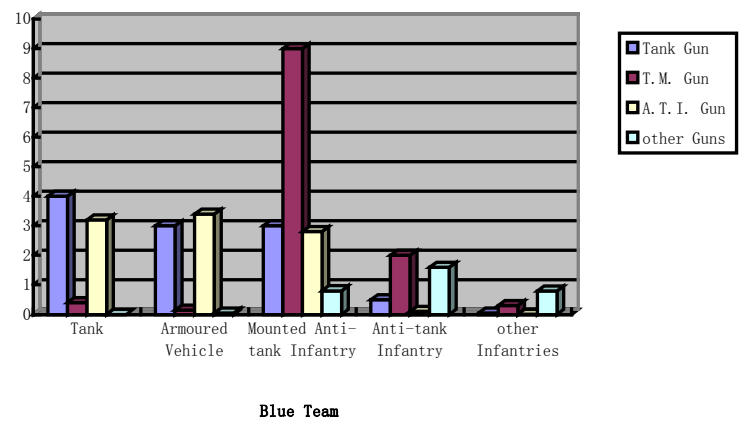

FIGURE VIII
In addition, there are four simplifications in this application: Firstly, some combat capability indicators of the equipment are not determined by combat tests, but are derived from empirical assumptions; secondly, some unconventional and non-mainbattle equipment are removed; Thirdly, the Agent's maintenance, medical and other supportive behavior are not considered; Fourthly, the battlefield environment and combat scenario design are not detailed enough.

\section{CONCLUSION}

The Agent model design of weapon equipment is the basis of modeling and combat simulation of SoS. The article chooses the automaton as the structural model of Agent, and divides the functional model of Agent into the combat ability model and the combat behavior model. Then, it designs the framework of Agent combat capability model for system simulation, and focuses on the construction method of Agent behavior model. Using the whole set of methods designed in this paper, we can build the Agent model of weapon equipment which meet the needs of system combat simulation. With a large number of Agent models of weapon equipment operating under the specific simulation environment, the emergent characteristics of the real system can emerge.

There are some shortcomings in the study which need to be solved in the future, such as the non-universality of the combat capability framework, and the failure of the Agent behavior modeling method when the simulated objects is non-cooperated or unable-cooperated.

\section{REFERENCES}

[1] Niu Xinguang. Defense system analysis of weapon equipment construction [M]. Beijing: National Defense Industry Press, 2007.

[2] Huang Jianxin. Research on System Effectiveness Simulation and evaluation method based on ABMS. [D]. National University of defense technology, 2011.

[3] A I. Irreducible Semi-Autonomous Adaptive Combat (ISAAC):An Artificial-Life Approach to Land Combat, Alenandria,Virginia: Center for Naval Analyses , 1997.

[4] Honabarger J B. Modeling Network Centric Warfare(NCW) with the SEAS[D]. Air Force Institute of Technology, 2006.

[5] W H C, I S. Complexity-Based Modeling and Simulation: Modeling Innovation at the Edge of Chaos[Z]. Monterey,California: 1998.

[6] Wang Jianping, Huang Kedi. Construction method of weapon equipment system model [J]. Journal of systems simulation, 2007 (05): 969-973.

[7] S C, L K, A T. Intelligent Agent for Aircraft Combat Simulation[Z]. Orlando,Florida: 1996. 